\title{
LA FORMACIÓN EN COMPETENCIAS PARA EL PROGRAMA DE TRABAJO SOCIAL EN LA UNIVERSIDAD DE LA GUAJIRA: UN CAMINO ABIERTO A LA DIVERSIDAD Y LA EDUCACIÓN
}

\author{
TRAINING IN COMPETENCIES FOR THE SOCIAL WORK PROGRAM AT THE \\ UNIVERSIDAD DE LA GUAJIRA: AN OPEN WAY TO DIVERSITY AND EDUCATION
}

\author{
Alma Luisa Fonseca-Mendoza' ${ }^{\text {ID }}$, Nicolás Tolentino Amaya-López² iD y Leonildes Astrid Gómez-Martínez ${ }^{\text {iD }}$
}

\begin{abstract}
RESUMEN
El presente estudio tiene como objetivo caracterizar, desde un enfoque de formación por competencias, el proceso formativo del programa de Trabajo Social en la Universidad de La Guajira. Para esto se profundiza en los lineamientos teóricos donde la formación por competencias genéricas y específicas en educación superior responda a las habilidades y vida productiva del estudiante y a las necesidades de un contexto determinado y enriquecido por sus condiciones culturales. La metodología abordada es de tipo etnográfico, con un diseño basado en la triangulación de la información. Los resultados indican que se requiere un nuevo planteamiento en la formación de competencias en el programa de Trabajo Social, donde se articulen los procesos curriculares, investigativo y la organización de las actividades académicas para que se evidencien mejores resultados en las pruebas saber y en la calidad educativa de la Universidad de La Guajira. Lo anterior pone en evidencia la necesidad de intervenir e investigar los factores asociados a la formación por competencias de los estudiantes, por cuanto el proceso formativo debe estar acorde con los requerimientos disciplinares, investigativos, profesionales, sociales, laborales y, por supuesto, tener como referente obligado el contexto sociocultural, porque es allí donde ocurren los imaginarios sociales y las prácticas en cotidianidad.
\end{abstract}

Palabras clave: Formación; competencias; Trabajo Social; contexto sociocultural; educación superior.

\begin{abstract}
The purpose of this study is to characterize the training process of the Social Work Program at the Universidad de La Guajira (Colombia), from a competency-based training approach. The theoretical guidelines state that training by means of generic and specific competences in higher education responds to the students skills and productive life as well as to the needs of a given context. And that such training is enriched by their cultural conditions. The methodology is ethnographic, with a design based on the triangulation of information. The results indicate that a new approach is required for the training of competences in the Social Work Program, where the curricular processes are articulated to research and to the organization of the academic
\end{abstract}

Fecha de recepción: julio 27 de 2016 / Fecha de aceptación: marzo 27 de 2017 / Publicado en línea: abril 19 de 2017 Tipología: Artículo de Investigación Científica y Tecnológica

Para citar este artículo: Mendoza, F. A., Amaya, L. N. \& Gómez, M. L. (2017). La formación en competencias para el programa de Trabajo Social en la Universidad de La Guajira: un camino abierto a la diversidad y la educación. Praxis, 13(1), 47 - 55. Doi: http://dx. doi. org/10.21676/23897856.2067

1. Mgs. en Educación de la Universidad de La Guajira. Correo electrónico: almaluisa@uniguajira.edu.co ORCID: 0000-0003-4892-1784

2. Ph.D. en Ciencias Mención Gerencia. Docente de la Universidad de La Guajira. Correo electrónico: namaya@uniguajira.edu.co 0RCID: 0000-0002-9760-1579

3. Mgs. en Educación de la Universidad de La Guajira. Correo electrónico: lagomez@uniguajira.edu.co ORCID: 0000-0002-1645-0511 
activities so that they end in better results in state tests and in the improved quality of education at the Universidad de La Guajira. The above, points to the need to intervene and investigate the factors associated with training by competences, since the training process must be in accordance with disciplinary, research, professional, social and work requirements and, of course, with the sociocultural context, because it is there that social imaginaries and practices in everyday life occur.

Keywords: Training; competences; Social Work; context sociocultural; higher education.

\section{INTRODUCCIÓN}

$\mathrm{E}$ n la actualidad, las universidades buscan Safanosamente el conocimiento. Por ende, son muchas las transformaciones que el entorno social y educativo ha tenido a raíz de la aparición de las nuevas tecnologías de las informaciones y las comunicaciones. Por tanto, las formas innovadoras exigen un maestro que esté preparado de manera integral para enfrentarse a los retos de la educación, lo que requiere mejorar los procesos formativos que lo caracterizan.

En este contexto y según la normatividad colombiana la educación universitaria está llamada a desarrollar, en su proceso formativo, las competencias en los estudiantes, por ello el decreto 1075 de 2015, promueve esta condición para responder a los desafíos que la educación se propone hoy. En consecuencia, las instituciones universitarias que han adoptado un enfoque formativo por competencias exploran nuevas opciones para facilitar el cambio en el sistema educativo, impactando con ello a nivel interno y externo de las instituciones y por ende en la calidad educativa. En palabras de Guglietta (2011), es este un reto que los formadores no pueden ni deben eludir; asumirlo con alguna garantía de éxito requiere hacer una revisión del rol como institución, apropiarse de los fundamentos del aprendizaje (constructivismo), de las herramientas y recursos disponibles (competencias informáticas e informacionales) y combinarlos en el diseño de estrategias educativas que promuevan el desarrollo de competencias de autogestión en los estudiantes.

Ligado a lo anterior, como proceso innovativo en los últimos años se ha observado que desde el Ministerio de Educación Nacional de Colombia, la incorporación de las competencias en el contexto curricular de la educación superior como enfoque, ha sido una iniciativa de la Educación Superior bajo una mirada integral del conocimiento, acentuando la formación con claros componentes transversales referidos a las competencias genéricas y específicas e incorporando propedéuticas como estrategias de enseñanza distintos a los utilizados tradicionalmente.

En atención a lo anteriormente citado, el proyecto educativo institucional de La Universidad de La Guajira considera:

El Perfil del egresado en ámbitos posibles, ubicando a la educación superior como proceso permanente que posibilita el desarrollo de las potencialidades del ser humano de manera integral, el cual, además, tiene como propósito generar transformaciones en la sociedad a través de la construcción de conocimientos y difusión de valores para la cualificación social. (Universidad de La Guajira, Proyecto educativo Institucional 2005, p.37).

El programa nombrado como Trabajo Social, objeto de la presente investigación, se creó y aprobó, cumpliendo con las normas legaes vigentes que van desde la Constitución Política de Colombia de 1991, la Ley 30 de 1992 que organiza el sistema de educación superior, la Ley 115 de 1994, la Ley 1188 de 2008, el Decreto 1295 de abril de 2010 .

Considerando lo descrito, el informe de Autoevaluación del programa de Trabajo Social con fines de Acreditación (2015), el documento es muy revelador de la situación del programa; los resultados de este proceso ofrecen información sobre los indicadores de opinión que 
tienen los estudiantes, docentes, administrativos, egresados y el sector productivo, en torno al quehacer de la institución en general y de este programa en particular, donde los profesores consideran en un $25 \%$ estar medianamente de acuerdo con que el currículo del programa reúne las condiciones de integralidad y calidad. En un $5 \%$, consideran no estar de acuerdo con esta afirmación.

Por su parte, los estudiantes, en un $40 \%$ expresan en el informe de autoevaluación, no conocer el proyecto educativo del programa, indicando además, en un $49 \%$, que en el programa en el que estudian no se desarrollan las competencias necesarias para lograr un buen desempeño profesional. En cuanto a los egresados se refiere, los datos indican en un $100 \%$ estar habilitados para desempeñarse en docencia, investigación, y como promotores comunitarios.

Con lo anteriormente descrito, se puede evidenciar que en general hay desconocimiento de parte de los docentes y estudiantes de lo contemplado en el proyecto educativo del programa, dejando entrever el poco conocimiento del documento y las dificultades para darle aplicabilidad a las competencias plasmadas en el trabajo anteriormente citado.

Si bien las competencias deben enfocar los aprendizajes, los aspectos específicos del personal docente, su accionar y el sistema evaluativo; el documento maestro del programa de trabajo social hace énfasis en las prácticas como base de los ejercicios de investigación, así mismo, desde esta perspectiva, se justifica la comprensión de los procesos psicosociales desde una perspectiva sociocultural.

Lo anterior, pone en evidencia la necesidad de intervenir en las competencias de los estudiantes por cuanto el proceso formativo debe estar acorde con los requerimientos disciplinares, investigativos, profesionales, sociales laborales y del contexto donde ocurre el programa.

En este marco de ideas se ubica el propósito de la investigación, es caracterizar, desde un enfoque de formación por competencias, el proceso formativo del programa de Trabajo Social en la Universidad de La Guajira. Se describen las competencias que desarrollan los estudiantes en el Proceso formativo del programa de Trabajo Social de la Universidad de La Guajira, también se Identifican los principales problemas del entorno social que debe saber orientar un Trabajador Social, se determinan las fortalezas y debilidades en el Proceso formativo. Finalmente se proponen lineamientos teóricos orientados a fortalecer las competencias en el Proceso formativo del programa de Trabajo Social de la Universidad de La Guajira.

\section{METODOLOGÍA}

Dado que el interés del estudio es conocer lo que piensan los estudiantes y docentes del proceso formativo del programa de trabajo social de la Universidad de La Guajira, se considera que el tipo de estudio es cualitativo y etnográfico, que busca la reflexión sobre las prácticas humanas que son susceptibles de investigar.

Según lo anterior, la orientación metodológica de la investigación cualitativa es comprender e interpretar la realidad tal como es vivida por las personas que participan en el contexto del estudio, por tal motivo se dará importancia a lo que los estudiantes y docentes piensan y practican en la realidad educativa de la Facultad de ciencias sociales y humanas y más a lo que se produce en este contexto universitario llamado Universidad de La Guajira.

La presente investigación consiste en describir detalladamente las situaciones, eventos, interacciones y comportamientos de los estudiantes y docentes, al decir de Murillo \& Martínez (2010) buscando "sus experiencias, actitudes, creencias, pensamientos y reflexiones tal como son expresadas por ellos mismos y no como el investigador quiere describirlo (p. 45). Por su parte, Hernández (2007) señala que: "hacer el estudio de forma holística y referida a un contexto cultural” (p.47).

De igual forma, se recurre a diferentes estrategias e instrumentos, así como a perspectivas teóricas para comprender la formación por competencias. 
De acuerdo con Pérez (2005), la investigación etnográfica describe la interpretación de lo que las personas hace, dicen, piensan y lo que significa para ellos lo que hacen.

Por lo tanto, se pretende describir e interpretar los fenómenos que ocurren en el programa de trabajo social de la universidad desde la perspectiva de los participantes, se refiere a la visión u orientación desde el interior y exterior del contexto educativo.

A través del proceso etnográfico, la fundamentación es holística y naturalista, ya que recoge una visión global del ámbito educativo, estudiado desde distintos puntos de vista: un punto de vista interno (estudiantes y docentes) y una perspectiva externa (la interpretación del investigador). Además, se destaca en el estudio el carácter de la investigación inductiva, Al respecto, Pérez (2005) afirma que "se basa en la experiencia y la exploración de primera mano sobre un escenario educativo a través de la observación participante como principal estrategia para obtener información” (p 84).

\section{Selección del diseño}

El diseño abordado es etnográfico porque existe flexibilidad en la utilización de estrategias como proceso de investigación. Al decir de Hernández (2007), "la investigación etnográfica busca captar la cultura (significados, normas, valores y puntos de vista) propios del contexto investigado" (p.97). Por lo tanto, las fases en el proceso de la presente investigación siguen lo dispuesto por Murillo \& Martínez (2010) quienes las describen como: “¿Qué es lo que quiero estudiar? ¿Cuál es mi objetivo? y cuál es el método que más se adapta a las respuestas que se buscan” (p.74). En otras palabras, lo que resulta preciso antes de iniciar la investigación referida a la caracterización del proceso de formación profesional de los estudiantes de Trabajo Social de la Universidad de La Guajira. Una mirada desde el enfoque de competencia de corte etnográfico.

\section{RESULTADOS Y DISCUSIÓN}

\section{Categorías, análisis e interpretación}

Con el propósito de ordenar los resultados obtenidos por objetivos, se ha organizado el análisis en varias partes. En la primera se presenta la información ya organizada producto de la discusión de los diferentes grupos focales con docentes y estudiantes del programa de Trabajo Social; de igual forma, la postura de los investigadores según la respectiva triangulación y teorización.

\section{Interpretación de los resultados: categoría competencias}

A continuación se realiza la triangulación para los actores docentes y estudiantes teniendo en cuenta las categorías competencias básicas, (interpretación de textos, comunicación verbal y escrita); de igual forma se toman las categorías competencias genéricas (relaciones interpersonales); y por último la categoría competencia específica en investigación y desde una postura hermenéutica de los investigadores.

Basado en las competencias básicas, según Tobón (2006), “el pensamiento lógico-matemático y las habilidades comunicativas (lenguaje) son la base para la apropiación y aplicación del conocimiento científico previsto para las distintas disciplinas sociales" (p.34). Las cuales se especifican en la interpretación de textos, la capacidad de leer, la comunicación verbal, escrita y del sistema numérico. Se puede afirmar que según el estudio realizado se encontró que hay mayor relevancia según el concepto de los informantes en la interpretación de textos y en la capacidad de leer, constituyéndose en un aspecto de gran importancia para la formación profesional en el programa de Trabajo Social.

De igual forma, Tobón (2006), conceptualiza las competencias genéricas como comunes a 
varias ocupaciones o profesiones, ellas hacen referencias a las relaciones interpersonales, a la responsabilidad, la autoestima, la creatividad y al saber. Como se puede observar en el presente estudio, hay mayor relevancia en las relaciones interpersonales y en la responsabilidad, puesto que son las más necesarias y sentidas según los diferentes grupos focales en el programa de Trabajo Social.

El mismo autor, Tobón (2006), conceptualiza sobre las competencias específicas como propias de una determinada ocupación o profesión, tienen un alto grado de especialización, encontrándose que ellas se encuentra inmersas en los procesos de investigación, la intervención, el diseño del programa, el seguimiento y la evaluación permanente de metas. Según el análisis realizado por los investigadores se encontró mayor relevancia en la investigación y en los diseños, posiblemente por la complejidad en su abordaje, según la mirada de los diferentes grupos focales, y la importancia que tiene la investigación como base transversal en el proceso formativo de la profesión de Trabajo Social.

Desde las competencias señaladas, se prevé el acto de las mismas, al integrar las siguientes; saber ser, saber hacer, saber conocer y saber convivir, con ello el trabajador social presenta un primer recurso para resolver problemas con creatividad, comprensión y emprendimiento, donde se muestra en escena niveles superiores de pensamiento, metacognitivo para el mejoramiento continuo y compromiso ético.

Lo anterior, según Tobón (2006):

"Está orientado por un dominio y un acumulado de experiencias de distintos tipos, lo que le ayuda al sujeto a desenvolverse en la vida práctica y a construir un horizonte social en relación con el otro para la configuración permanente del ser” (p. 39).

De igual forma, en lo expresado por el CONETS (2008):

Refleja la capacidad que tiene el trabajador social para comunicarse de manera eficaz en contextos específicos, donde el punto de partida es la acción, como un conjunto de procesos y conocimientos para interpretar, argumentar o producir discursos pertinentes a la situación y contexto en la cual se establece la relación. (p.27).

Se infiere entonces, según el Concejo Nacional de Trabajo Social (2008) (CONETS), que:

la importancia de lograr la capacidad que tiene una persona para comunicarse de manera eficaz en contextos específicos, para lo cual, en este caso el punto de partida es la existencia de personas que ponen en acción un conjunto de procesos y conocimientos para interpretar, argumentar o producir discursos pertinentes a la situación y contexto en la cual se establece la comunicación. (p. 32)

Se requiere en el proceso formativo una mayor capacidad en el estudiante para desempeñar otras funciones ya no solo en manejo de conflictos familiares. Se debe ser más productivo y en diferentes contextos. Esto contradice un poco con lo dispuesto por el Ministerio de Educación Nacional (2015), donde en sus guías de competencias, establece que:

Tener competencia es usar el conocimiento para aplicarlo a la solución de situaciones nuevas o imprevistas, fuera del aula, en contextos diferentes, y para desempeñarse de manera eficiente en la vida personal, intelectual, social, ciudadana y laboral. Las competencias que el sistema educativo debe desarrollar en los estudiantes son de tres clases: básicas, ciudadanas y laborales. (p. 17).

Por lo anterior no se vislumbra claramente en el proyecto educativo del programa de Trabajo Social la competencia que designe básicamente la aplicación de un conocimiento complejo en contexto; que expresa la capacidad del estudiante de poner el conocimiento en transferencia, es decir un conocimiento que pueda atender las problemáticas del contexto.

Las pruebas SABER Pro son reveladoras, los estudiantes del trabajo cual no cumplen con las expectativas, recordemos que anteriormente se dijo que los resultados reflejan un bajo nivel y por debajo de la norma nacional. Se debe tener dominio en diversas áreas de intervención, como también poseer conocimiento en formas de intervención para atender así mismo otras situaciones diferentes a los conflictos familiares. 
Para resaltar, es importante analizar las relaciones interpersonales como una de las competencias básicas de mayor abordaje en el programa de Trabajo Social, esto le ayuda a las personas a tener mayor impacto social y a mirar otras opciones de abordaje, ya que la interacción con los otros hace que pueda tener habilidad para identificar problemas y resolverlos.

Por otra parte, según los actores docentes y estudiantes, los problemas del entorno social que debe saber orientar un trabajador social en el proceso formativo son precisamente los conflictos, determinando así mismo, a la familia como área de mayor intervención profesional del trabajador social.

En atención a lo descrito anteriormente, en el documento maestro del programa de Trabajo Social hay unas áreas de intervención las cuales están derivadas de la siguiente forma: la familia, comunidad, organización, educación y rehabilitación. Según los resultados encontrados, la familia es el área de más relevancia para los docentes y estudiantes. Al respecto, la familia, según las Organización de las Naciones Unidas, es el núcleo fundamental de la sociedad. En segundo lugar aparece el área comunitaria como fundamental en toda intervención que conlleve a alguna problemática existente como principal abordaje para el Trabajador Social.

Al respecto, según Mejía (1998) "la profesión de Trabajo Social interviene en diversos espacios denominados áreas, a las que el mencionado autor la denominan campos relacionados a la intervención de Trabajo Social en familia” (p.45), en donde también la profesión tiene muchas posibilidades de actuación.

Para el Trabajo Social es indispensable concebir que la persona se encuentra dispuesta al cambio social, además del político, económico y cultural en "un instante en particular de su vida, entendido como el ciclo vital.” (Hernández, 1997, p.28). En su libro, Familia, ciclo vital y Psicoterapia Sistémica, la define en el ciclo vital como: "La unidad básica de la sociedad con base en la pareja conyugal, donde se desarrollan valores, objetivos afines y una cultura que les permite la adaptación del individuo a la organización social” (p. 68).

En este sentido, la familia tiene por objetivo la reproducción, donde cada miembro tiene derechos y deberes, contextualizada como una institución social y gestora de elementos culturales. Según González (2008):

El conjunto de relaciones emocionales y el ser humano pasa por etapas reguladas por una sucesión de ciclos que se asocian a la edad o a una serie de derechos y obligaciones, basados en la intervención que se realiza desde la familia. (p.69).

Según lo anterior, los principales problemas sociales que debe saber orientar un profesional de Trabajo Social y atendiendo al contexto en la Guajira es la desnutrición infantil, violencia intrafamiliar, contrabando y sicariato. Pero esto contrasta con lo descrito anteriormente, encontrando la violencia intrafamiliar como el principal componente que transforma las relaciones entre los miembros de una misma familia, causando efectos físicos, emocionales, sexuales o económicos. Dentro de este enfoque, la Organización Mundial de la salud (OMS), para la prevención de la violencia intrafamiliar, determina como principal factor, fomentar en las familias formas de resolución de conflictos.

Según los resultados encontrados, se encontró la violencia intrafamiliar como la problemática más relevante hallada en el contexto guajiro, dicho de esta manera se puede afirmar que el Trabajador Social cumple un papel importante dentro de la sociedad como también lo es el rol de investigador familiar como principal motor de la sociedad. Al respecto, el trabajador social en la Universidad de La Guajira contempla en su currículo el Trabajo Social en comunidad, orientado al cambio, al desarrollo comunitario, pero se observa el interés del programa en la investigación de la familia y en los conflictos hacia la acción social con fines de ajuste, desarrollo o transformación.

Lo anterior, deja ver que hay poca atención en la construcción de condiciones para la organización y el desarrollo de la comunidad, pero con mucho interés hacia los procesos de investigación social, 
encontrándose una inconsistencia en lo observado, por un lado lo que asumen los docentes y estudiantes y por otra parte lo encontrado en el plan de estudio. El plan de estudio presenta un marcado abordaje de los métodos y procesos de investigación, pero no refleja el énfasis en la familia ni en los conflictos sociales.

El Concejo Nacional de Trabajo Social, CONETS, como máxima representación que direcciona la formación del trabador social indica que existen hoy múltiples formas de ser, conocer y hacer Trabajo Social, alimentadas por enfoques epistemológicos y teóricos diversos. En este sentido, el CONETS (2008) afirma: "Se ha permitido que en la profesión se gesten desarrollos por áreas específicas de intervención, que han dado avances en espacios particulares de la praxis profesional y a la emergencia de diferentes sectores, campos, niveles y enfoques de la misma” (p.41).

Estos avances de la profesión han suscitado debates muy interesantes alrededor del objeto de estudio de la profesión.

En cuanto a las fortalezas y debilidades en el Proceso formativo del programa de Trabajo Social de la Universidad de La Guajira, los actores como docentes y estudiantes consideran el componente de investigación como principal fortaleza en el proceso formativo y como principal debilidad la distribución del trabajo académico, seguido del plan de estudio.

Según el análisis realizado, los grupos focales consideran que el componente investigación y la variedad de metodologías en el proceso formativo se constituyen en la fortaleza más importante del programa de Trabajo Social. De igual forma, consideran que en términos de debilidad se encontraron en primer lugar la distribución del trabajo académico y en segundo lugar el plan de estudio. Al decir de los informantes, existen espacios limitados y recarga de información durante el proceso académico del semestre.

Como fortaleza se destaca en los resultados la categoría de investigación, reflejado en las políticas, estrategias y recursos para la investigación, así como el ambiente para una cultura investigativa en la institución y el programa. En este aspecto, el plan de estudio del programa presenta un énfasis marcado en la investigación, toda vez que los estudiantes desde el primer semestre inician con cursos de metodologías y de diseños investigativos. Lo anterior también se corrobora con los resultados de las pruebas saber, donde los percentiles en investigación son los que más sobresalen.

Si bien la investigación es la fortaleza, no se refleja en los resultados de las pruebas un alto nivel en los procesos de intervención del profesional de trabajo social propuesto por el CONETS como ente rector de direccionamiento en competencias para los programas de Trabajo Social en Colombia. Sin embargo, este organismo, propone la identificación, preparación y la realización de intervenciones sociales apropiadas para conseguir las competencias requeridas para un Trabajador Social.

Por otra parte, los propósitos de formación no se ven en coherencia con las competencias que describen los informantes como las más destacadas en el programa. En el plan de estudios se presentan cursos y asignaturas que en esencia son relevantes, pero no se observa como éstas se articulan de forma coherente con las competencias que se muestran de interés en los documentos institucionales, o al menos no hay un documento que pueda promover la coherencia y hacer esa relación, es decir, un plan de estudios en coherencia con las competencias, como lo indica el Decreto 1075 de 2015.

Por otro lado, en el desarrollo de la experiencia y su posterior sistematización y evaluación, hacen pensar en un programa basado en una formación con un claro componente investigativo, donde la creatividad y el análisis crítico es lo que conjuga las competencias formativas en la formación del Trabajador Social de la Universidad de La Guajira. Se propone que la investigación en Trabajo Social sea aplicada, orientada a potenciar cambios sociales y a la trasformación de la sociedad y la familia. 


\section{CONCLUSIONES}

Ante los nuevos enfoques epistémicos, producto de la modernización, el desarrollo económico, la democratización y el impulso de la tecnología, el Trabajo Social se debe a la presentación de una intervención destinada a la integración sistemática y basada en la reducción de los conflictos sociales y sus derivados. La propuesta de intervención está basada en unos objetivos de integración social y al fortaleciendo las diferencias ante las distintas disfunciones, sobre todo en las familias para dar respuestas a los conflictos y desviaciones sociales.

Se resalta en el proceso investigativo un programa de Trabajo Social con alto sentido de cientificidad donde se asume la ciencia desde la interdisciplinariedad. Se requiere así mismo, planes de estudios basados en competencias para la formación teórica (apropiación del conocimiento) y las metodologías (técnicas de intervención e investigación) sobre problemáticas relacionadas con las distintas disfunciones sociales. Se propone entonces, una propuesta basada en las competencias que dé respuesta al problema social, político, económico y sociocultural que atraviesa Colombia y la Guajira en particular. Se debe contar con planes de estudio ajustados a la realidad social y que además, las condiciones logísticas y recursos bibliográficos correspondan a la comprensión y análisis del contexto donde se desarrolla el proceso formativo.

Por otra parte, se requiere del desarrollo de competencias orales y escritas como imperativa sustancial para el Trabajo Social. La puesta en marcha de un binomio teoría y práctica en trabajo social favorece el espíritu creativo y el aprender a hacer. En este sentido, se reconoce que las funciones cognitivas superiores se activan si se enfrenta el conocimiento a situaciones y problemas para la posterior conceptualización y despliegue de respuestas definidas. De igual forma, el aprender a ser, es específicamente permitir que el estudiante promueva la personalidad y el sentido crítico en el abordaje de campos y núcleos problémicos referidos a la intervención social.
Elaborar el plan de re contextualización de la formación donde el docente y estudiante sean la guía para promover cambios en los planes de estudios, métodos, didácticas y prácticas profesionales. Se requiere una redefinición en la formación del trabajo social, una visión, una estructuración donde se caractericen las problemáticas existentes, y nuevas propuestas en los contenidos programáticos, métodos y procedimientos de enseñanza basados en competencias.

La formación del estudiante debe articular los requerimientos de la sociedad, es decir, lo que la sociedad necesita. Articular la renovación como un condicionante para la acreditación del programa de Trabajo Social, los planes de estudios y la práctica docente. Lo anterior, se debe reorientar de acuerdo con las circunstancias sociales e históricas de Colombia y el Departamento de la Guajira.

Por otra parte, se requiere de una reorganización administrativa, la administración es un escape para lograr el éxito en la formación profesional. "un acto pedagógico basado en competencias".

Según los resultados, la formación del trabajador social debe actualizarse en aspectos como la cultura general, metodologías, ciencias humanas, estudios de problemas contemporáneos y un mayor adiestramiento en los problemas básicos del contexto. Se requiere un modelo de Trabajo Social basado en un sistema educativo con un perfil político - pedagógico y en concordancia con una formación permanente.

Se propone, a partir del análisis, nuevos desafíos en el programa de Trabajo Social de la Universidad de La Guajira, un énfasis para la intervención, que diferencie la representación de modernidad y modernización.

\section{REFERENCIAS BIBLIOGRÁFICAS}

Concejo Nacional de Trabajo Social, Conets, (2008). Marco de fundamentación conceptual en Trabajo Social. Medellín. Recuperado de http://www.goglee.com.co/searchq:conets2 008marcodefundamentaciontrabajosocialmedellin@oq 
Decreto 1075 de 2015, (Mayo 26). Por medio del cual se expide el Decreto Único Reglamentario del Sector Educación Recuperado de http:// www.mineducacion.gov.co/1759/w3-article-351080.html

González, A. (2008). La familia. Una mirada desde la Psicología. Medisur, 6(1), 4-13.

Guglietta, M. (2011). Educación superior por competencias, constructivismo y tecnologías de la información y las comunicaciones (TIC). Una visión integrada. Recuperado de http:// www.iesalc.unesco.org.ve/index.php?... competencias-constructivismo

Hernández, R. (2007). Metodología de la investigación. México: Mac Graw Hill.

Hernández, A. (1997). Familia, ciclo vital y Psicoterapia Sistémica. Bogotá: El Búho.

Ministerio de Educación Nacional decreto $\mathrm{N}^{\circ} 1295$ República de Colombia 20 de abril de 2010. Por el cual se reglamenta el registro calificado que trata la Ley 1188 de 2008 y la oferta y desarrollo de programas académicos de educación superior. Recuperado de http://www.mineducacion.gov.co/1621/ articles-229430_archivo_pdf_decreto1295

Mejía, J. (1998). El Trabajador Social de cara al futuro, visión prospectiva. Revista de TS, (12). Cali: CONETS, FECTS.

Murillo, C., \& Martínez, C. (2010). Métodos de Investigación Educativa en $3^{\circ} \mathrm{Ed}$. Especial.

Organización Mundial de la Salud (2005): Estudio multipais de la OMS sobre la salud de la mujer y violencia domestica contra la mujer: primeros resultados sobre prevalencia, eventos relativos a la salud y respuesta de las mujeres a dicha violencia: resumen del informe. Suiza. Pérez, A. (2005). Métodos de investigación en educación. Universidad autónoma de Madrid.

Tobón, S. (2006). Aspectos básicos de la formación basada en competencias. Proyecto Talca Mesesup.

Universidad de La Guajira (2005). Proyecto educativo Universitario. Recuperado de http:// www.uniguajira.edu.co 